\title{
A broadband multi-distance approach to measure tissue oxygen saturation with continuous wave near-infrared spectroscopy
}

Kovácsová, Zuzana, Bale, Gemma, Veesa, Joshua Deepak, Dehghani, Hamid, Tachtsidis, llias

Zuzana Kovácsová, Gemma Bale, Joshua Deepak Veesa, Hamid Dehghani, Ilias Tachtsidis, "A broadband multi-distance approach to measure tissue oxygen saturation with continuous wave near-infrared spectroscopy," Proc. SPIE 11074, Diffuse Optical Spectroscopy and Imaging VII, 110740P (11 July 2019); doi: 10.1117/12.2527180

SPIE. Event: European Conferences on Biomedical Optics, 2019, Munich, Germany 


\title{
A broadband multi-distance approach to measure tissue oxygen saturation with continuous wave near-infrared spectroscopy
}

\author{
Zuzana Kovácsováa ${ }^{\text {, Gemma Bale }}$, Joshua Deepak Veesa ${ }^{\mathrm{b}}$, Hamid Dehghani $^{\mathrm{b}}$, and Ilias \\ Tachtsidis $^{\text {a }}$ \\ aDepartment of Medical Physics and Biomedical Engineering, University College London, \\ London, UK \\ ${ }^{\mathrm{b}}$ School of Computer Science, University of Birmingham, Birmingham, UK
}

\begin{abstract}
Brain tissue oxygen saturation, $\mathrm{StO}_{2}$, measured with near-infrared spectroscopy (NIRS) is of great clinical interest as it quantifies the balance between cerebral oxygen supply and demand. Some brain oximeters are based on spatially resolved spectroscopy (SRS), where NIRS data is collected at multiple distances from the light source to estimate a slope of light attenuation against distance. Other use a broadband approach which utilizes derivatives of the absorption spectra to estimate $\mathrm{StO}_{2}$, such as broadband fitting (BF). We describe a novel algorithm, broadband spatially resolved spectroscopy (BB-SRS), for estimating $\mathrm{StO}_{2}$. It is based on comparing the measured slope to a model of the attenuation slope, which depends on the optical properties of tissue. Fitting this model with a least squares fitting procedure recovers parameters describing absorption and scattering; the concentrations of oxy- and deoxy-haemoglobin and hence $\mathrm{StO}_{2}$ and the scattering parameters $\beta$ and $\alpha$ describing the exponential dependence of scattering on wavelength. To demonstrate BB-SRS, a broadband spectrum (700 $1000 \mathrm{~nm}$, step size $2 \mathrm{~nm}$ ) was simulated in NIRFAST and was analysed with BB-SRS, SRS and BF. The developed BB-SRS algorithm recovered $\mathrm{StO}_{2}$ with a relative error of $-9 \%$; the concentration of deoxyhaemoglobin with a relative error of $+4 \%$, oxyhaemoglobin $-10 \%$. The scattering parameters $\beta$ and $\alpha$ were recovered with a relative error of $-30 \%$ and $-2 \%$, respectively. Among the three algorithms, BB-SRS performed with the best relative error.
\end{abstract}

Keywords: brain oximetry, near-infrared spectroscopy, broadband

\section{INTRODUCTION}

Brain tissue oxygen saturation $\mathrm{StO}_{2}$ is defined as the ratio of oxygenated and total haemoglobin and provides a measure of the balance between oxygen supply and demand. Most continuous-wave (CW) near-infrared spectroscopy (NIRS) systems measure only chromophore concentration changes; $\mathrm{StO}_{2}$ measurement can be achieved with multi-distance (the use of more than one light source-detector separation) or broadband $(\sim 650-1000 \mathrm{~nm})$ algorithms. However, brain oximetry with CW NIRS is criticized for its lack of standardization and clinically insufficient precision. The motivation behind our work is whether we can improve the precision of the measurement by developing a more robust method to measure $\mathrm{StO}_{2}$ with CW NIRS by combining multi-distance and broadband algorithms, as each has its own benefits. The main strength of multi-distance setups is overcoming the layered structure of tissue, and broadband methods yield more spectroscopic information about tissue.

\subsection{Multi-distance Oximetry}

Spatially resolved spectroscopy (SRS) ${ }^{1}$ is used in commercial systems, e.g. NIRO systems. ${ }^{2}$ SRS uses multiple light source-detector separations to quantify the slope of light attenuation $A$ against distance $\rho, \partial A / \partial \rho$. Assuming that scattering is linearly dependent on wavelength, $\mu_{s}^{\prime}(\lambda)=k \cdot(1-h \cdot \lambda)$; where $h=0.00063 \mathrm{~mm}^{-1} \mathrm{~nm}^{-1},{ }^{1}$ it is possible to obtain a scaled attenuation coefficient $k \mu_{a}$ from the slope measurement, which then leads to $\mathrm{StO}_{2}$ :

$$
\mathrm{StO}_{2}=\frac{\left[\mathrm{HbO}_{2}\right]}{k[\mathrm{HH}]+k\left[\mathrm{HbO}_{2}\right]} \times 100 \%=\frac{\left[\mathrm{HbO}_{2}\right]}{[\mathrm{HH}]+\left[\mathrm{HbO}_{2}\right]} \times 100 \% .
$$

Diffuse Optical Spectroscopy and Imaging VII, edited by Hamid Dehghani, Heidrun Wabnitz, Proc. of SPIE-OSA Vol. 11074, 110740P · C 2019 SPIE-OSA · CCC code: 1605-7422/19/\$21 · doi: 10.1117/12.2527180 


\subsection{Broadband Oximetry}

Broadband CW NIRS can be used to measure $\mathrm{StO}_{2}$ with differential analysis, ${ }^{3}$ which will further be referred to as broadband fitting (BF). BF applies only one light source-detector separation and a broadband spectrum $(\sim$ $650-1000 \mathrm{~nm}$ ). The idea of the algorithm is to compare the measured reflectance $R_{M}$ to a theoretical model $R_{T}$ (see Kienle A, 1997). ${ }^{4}$ The measured reflectance $R_{M}$ is fitted with the model in the first and second spectral derivative space; the fitting parameters describe scattering and absorption: $\beta$ and $\alpha$ from $\mu_{s}^{\prime}(\lambda)=\beta \lambda[\mu m]^{-\alpha}$; the other fit coefficients are chromophore concentrations; $\mu_{a}(\lambda)=\sum c_{N} \epsilon_{N}(\lambda)$, where $\epsilon$ is the specific extinction coefficient and $c_{N}$ is concentration of selected chromophores. Obtaining these parameters leads to $\mathrm{StO}_{2}$.

\section{METHODS}

The new method, broadband SRS (BB-SRS), is based on the same model as BF, $R_{T}$, and applies it to a multi-distance setting by using it to calculate a model of the slope of attenuation $\partial A_{T} / \partial \rho$. It is then fitted to the measured slope in the first spectral differential space similarly to BF, yielding information about both chromophore concentrations and scattering properties. The method has several benefits, such as eliminating errors caused by detector/tissue coupling and any other baseline offset by moving to the differential space, yielding more tissue properties than just $\mathrm{StO}_{2}$, and accounting for tissue layers.

The first testing of the method was performed on broadband data simulated with NIRFAST. ${ }^{5}$ Broadband $(700$ $1000 \mathrm{~nm}$, step size $2 \mathrm{~nm}$ ) intensity spectra at 2 light source-detector separation $(20,25 \mathrm{~mm})$ were generated on a homogeneous mesh model of a 40 - week neonatal head. ${ }^{6}$ The optical properties were set to gray matter (see Tab. (1)). ${ }^{7}$ The data were analysed with all three algorithms; SRS *, BF and BB-SRS.

\section{RESULTS}

Fig.(1) shows the individual steps in simulated spectrum analysis with BB-SRS, SRS and BF. Tab. (1) shows the $\mathrm{StO}_{2}$, chromophore concentrations and scattering properties obtained with the three different methods form the same data. SRS recovered $\mathrm{StO}_{2}$ with a relative error of $-46 \%$, BF with $7 \%$ and BB-SRS $-9 \%$. The four parameters $[\mathrm{HH} b],\left[\mathrm{HbO}_{2}\right], \beta$ and $\alpha$ were recovered with BF/BB-SRS with the following relative errors: $-51 \% / 3 \%,-36 \% /-10 \%, 20 \% /-30 \%$, and $54 \% /-2 \%$.

Table 1. Simulated broadband spectrum analysis results obtained with three different algorithms: spatially resolved Spectroscopy (SRS), broadband fitting (BF) and broadband SRS (BB-SRS). The number in the brackets is the difference between the true value and the result of the method. The true values are optical properties of grey matter. ${ }^{7}$

\begin{tabular}{|l|l|l|l|l|l|}
\hline & $\mathrm{StO}_{2}[\%]$ & {$[\mathrm{HHb}][\mu \mathrm{M}]$} & {$\left[\mathrm{HbO}_{2}\right][\mu \mathrm{M}]$} & $\beta\left[\mathrm{mm}^{-1} \mathrm{~nm}^{-1}\right]$ & $\alpha[-]$ \\
\hline True value & 60.75 & 38.40 & 54.80 & 0.98 & 0.61 \\
\hline SRS & $32.87(-27.88)$ & $/$ & $/$ & $/$ & $/$ \\
\hline BF & $65.12(+4.37)$ & $18.70(-19.70)$ & $34.91(-19.89)$ & $1.18(+0.20)$ & $0.94(+0.33)$ \\
\hline BB-SRS & $55.32(-5.43)$ & $39.87(+1.47)$ & $49.36(-5.44)$ & $0.69(-0.29)$ & $0.60(-0.01)$ \\
\hline
\end{tabular}

\section{SUMMARY}

We present a novel approach to measuring brain tissue oxygen saturation with NIRS based on modelling the slope of attenuation against distance in tissue. The algorithm was demonstrated on simulated broadband data and compared to other brain oximetry algorithms, SRS and BF. SRS estimate of $\mathrm{StO}_{2}$ was the most different from the true value; $\mathrm{BF}$ was the closest - however, the recovered concentrations of oxy- and deoxy-haemoglobin are more different from the true values than those obtained with BB SRS. Overall, the relative error of BB-SRS was the smallest among the three algorithms and it provided the most accurate results. Further work is focused on

*As SRS has not been designed for broadband data, analysis was performed with selected wavelengths, 735, 810 and $850 \mathrm{~nm}$, which are wavelengths used in a commercial SRS device, NIRO 200NX. ${ }^{2}$ 
the improvement of scattering properties recovery and the application of BB-SRS to data collected in phantoms, adults and neonates.

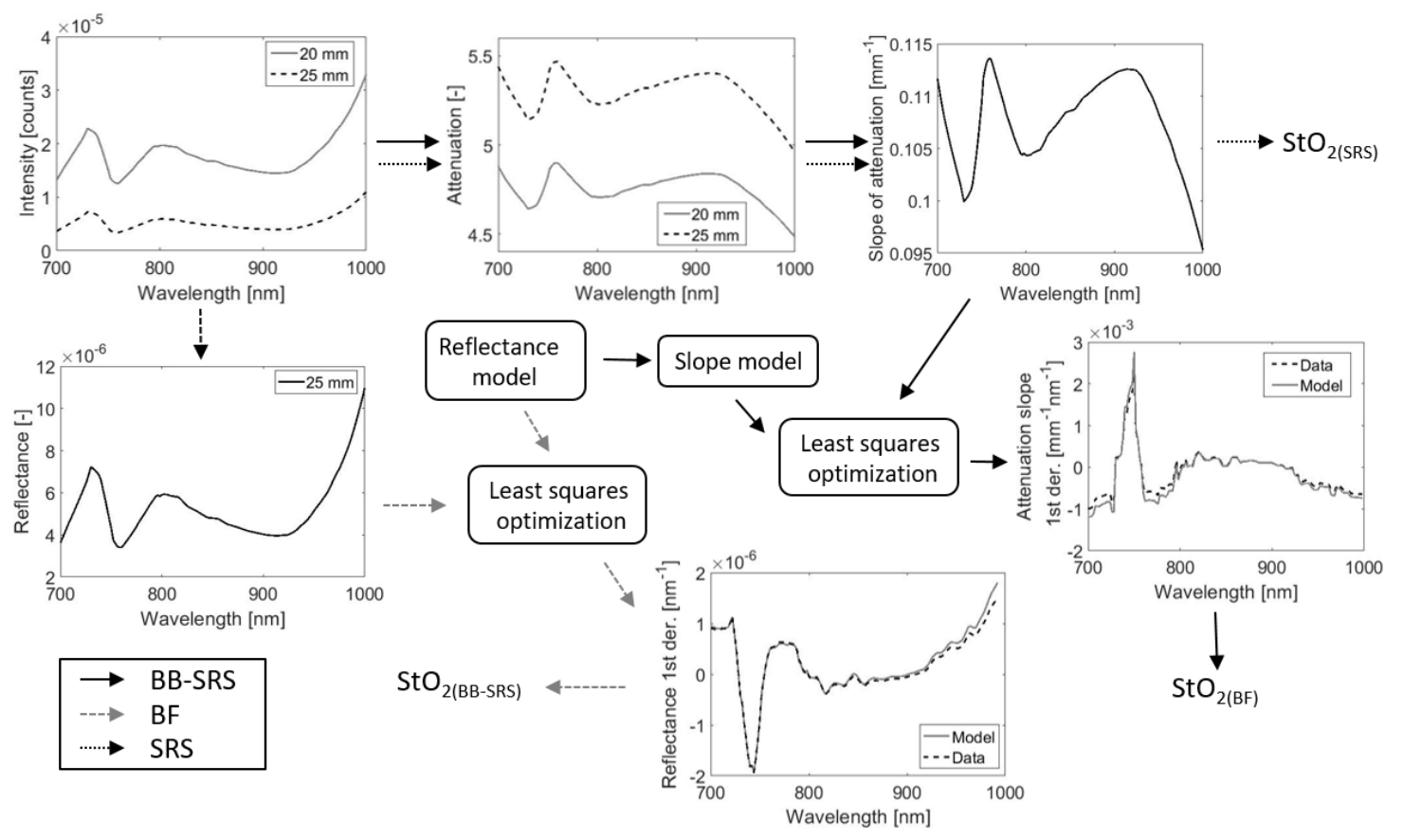

Figure 1. The process of calculating $\mathrm{StO}_{2}$ from the simulated broadband data with SRS (dotted arrows), BB-SRS (full arrows) and BF (dashed arrows). The reflectance model used can be found in Kienle A, $1997 .^{4}$

\section{ACKNOWLEDGMENTS}

This project has received funding from the European Union's Horizon 2020 research and innovation programme under the Marie Sklodowska-Curie grant agreement No 675332. IT is supported by The Wellcome Trust, grant 104580/Z/14/Z. GB is supported by the Medical Research Council, grant MR/S003134/1.

\section{REFERENCES}

[1] Suzuki, S., Takasaki, S., Ozaki, T., and Kobayashi, Y., "A tissue oxygenation monitor using NIR spatially resolved spectroscopy," SPIE Proceedings 3597, 582-592 (1999).

[2] "NIRO-200NX Near infrared oxygenation monitor C10448 - Hamamatsu Photonics."

[3] Yeganeh, H. Z., Toronov, V., Elliott, J. T., Diop, M., Lee, T.-Y., and St. Lawrence, K., "Broadband continuous-wave technique to measure baseline values and changes in the tissue chromophore concentrations," Biomedical Optics Express 3(11), 2761 (2012).

[4] Kienle, A. and Patterson, M. S., "Improved solutions of the steady-state and the time-resolved diffusion equations for reflectance from a semi-infinite turbid medium," J. Opt. Soc. Am. A 14(1), 246-254 (1997).

[5] Dehghani, H., Eames, M. E., Yalavarthy, P. K., Davis, S. C., Srinivasan, S., Carpenter, C. M., Pogue, B. W., and Paulsen, K. D., "Near infrared optical tomography using NIRFAST: Algorithm for numerical model and image reconstruction.," Communications in numerical methods in engineering 25, 711-732 (8 2008).

[6] Brigadoi, S., Aljabar, P., Kuklisova-Murgasova, M., Arridge, S. R., and Cooper, R. J., "A 4D neonatal head model for diffuse optical imaging of pre-term to term infants," NeuroImage 100, 385-394 (2014).

[7] Eggebrecht, A. T., White, B. R., Ferradal, S. L., Chen, C., Zhan, Y., Snyder, A. Z., Dehghani, H., and Culver, J. P., "A quantitative spatial comparison of high-density diffuse optical tomography and fMRI cortical mapping.," NeuroImage 61, 1120-8 (7 2012). 